\title{
Biweekly docetaxel, fluorouracil, leucovorin, oxaliplatin (TEF) as first-line treatment for advanced gastric cancer and adenocarcinoma of the gastroesophageal junction: safety and efficacy in a multicenter cohort
}

\author{
Simon Pernot • Emmanuel Mitry • Emmanuelle Samalin • Laetitia Dahan • Cécile Dalban • \\ Marc Ychou • Jean-François Seitz • Hajer Turki • Thibault Mazard • Aziz Zaanan • \\ Céline Lepère $\cdot$ Jean-Nicolas Vaillant $\cdot$ Bruno Landi $\cdot$ Philippe Rougier $\cdot$ Julien Taieb
}

Received: 16 January 2013/Accepted: 21 April 2013/Published online: 6 June 2013

(c) The International Gastric Cancer Association and The Japanese Gastric Cancer Association 2013

\begin{abstract}
Background Docetaxel-cisplatin-5-FU chemotherapy is superior to 5-FU-cisplatin in terms of response rate and survival in advanced gastric cancer (AGC), but is more toxic. Oxaliplatin is better tolerated than cisplatin, which it can effectively replace in this setting. We hypothesize that incorporating docetaxel into a simplified FOLFOX regimen should be a tolerable and effective option in first-line treatment of AGC.

Methods Data were collected at six French centers from patients with metastatic or local AGC who received docetaxel, fluorouracil, leucovorin, or oxaliplatin (TEF) as first-line treatment. TEF was administered as follows: docetaxel $\left(50 \mathrm{mg} / \mathrm{m}^{2}\right)$, oxaliplatin $\left(85 \mathrm{mg} / \mathrm{m}^{2}\right)$, and leucovorin $\left(40 \mathrm{mg} / \mathrm{m}^{2}\right)$ on day 1 , and 5-FU continuous infusion for $48 \mathrm{~h}\left(2400 \mathrm{mg} / \mathrm{m}^{2}\right)$ every 2 weeks.
\end{abstract}

\author{
S. Pernot $(\varangle) \cdot$ A. Zaanan · C. Lepère - J.-N. Vaillant · \\ B. Landi · P. Rougier · J. Taieb $(\square)$ \\ HEGP, APHP, Paris Sorbonne Cité, Université Paris Descartes, \\ Paris, France \\ e-mail: simon.pernot@gmail.com \\ J. Taieb \\ e-mail: jtaieb@club-internet.fr \\ E. Mitry \\ Institut Curie, St-Cloud, France \\ E. Samalin · M. Ychou \\ CRLC Val D'aurelle, Montpellier, France \\ L. Dahan - J.-F. Seitz $\cdot$ H. Turki \\ La Timone, Aix-Marseille Université, Marseille, France \\ C. Dalban \\ CLCC Georges François Leclerc, Dijon, France \\ T. Mazard \\ CHU de Montpellier, Montpellier, France
}

Results Forty-one patients were enrolled. Performance status was grade 0 and 1 in respectively 27 and $58 \%$ of patients; 17 patients had adenocarcinoma of the gastroesophageal junction; 37 patients had metastatic disease, 22 had a poorly differentiated or diffuse type. Objective response rate was $66 \%$, with a complete response in two patients $(5 \%)$. Median progression-free survival and overall survival were respectively 6.3 and 12.1 months. Tolerability was acceptable with no treatment-related deaths. The most frequent grade 3-4 toxicities were neutropenia (30\%) and neuropathy $(12.5 \%)$. Curative intent surgery after response to TEF was performed in seven patients $(17 \%)$.

Conclusion TEF is an effective first-line treatment with an acceptable toxicity profile for patients with AGC. It may allow curative resection in initially unresectable patients. TEF should now be evaluated in prospective randomized trials.

Keywords Docetaxel - Oxaliplatin · Gastric cancer

\section{Introduction}

Systemic chemotherapy of locally advanced or metastatic gastric adenocarcinoma is effective in term of responses rates, quality of life and survival time, but the benefits are of limited duration and tolerability varies according to the therapeutic protocol [1].

Several regimens have been developed, but there is no universal standard chemotherapy for these patients. In the European Society for Medical Oncology (ESMO) guidelines [2], an epirubicin (E), cisplatin (C) and 5-fluorouracil $(5-\mathrm{FU})$ regimen $(\mathrm{ECF})$ is the reference for palliative chemotherapy in advanced gastric cancer (AGC) [3-5]. However, 5-FU or capecitabine (X) and cisplatin (FP and $\mathrm{XP})$ combinations are likely equivalent in terms of efficacy 
and tolerability, as shown with a monthly FP [6] combination or with biweekly LV5FU2-P [7] or the XP combination [8]. The added value of using anthracyclines is not obvious, as shown in the GASTRIC meta-analysis [9].

The docetaxel (D)-cisplatin-5-FU (DCF) regimen has been shown to be superior to FP $[10,11]$ in a phase III trial, with an improvement in time to progression (HR: 1.47), overall survival (OS) and quality of life. However, DCF has not been widely used because it is associated with a high rate of hematological toxicity, including grade 3-4 neutropenia ( 82 vs. $57 \%$ ) and complicated neutropenia ( 29 vs. $12 \%$ ).

Attempts to improve the therapeutic index of the DCF regimen have been explored in many trials by lowering the doses, modifying the schedule or replacing 5-FU or cisplatin by better tolerated drugs. Capecitabine and oxaliplatin can advantageously replace IV 5-FU and cisplatin as shown in the Real 2 trial. In this study, in more than 1,000 patients, 12-month OS was observed in patients treated with EOX, compared with 9.9-month OS for patients treated with the old ECF schedule [12]. Oxaliplatin seems to be associated with less neutropenia but increased diarrhea. These results have been supported by another randomized trial in which oxaliplatin combined with 5-FU (FLO regimen) was as effective as a 5-FU, leucoverin and cisplatin combination (FLP) [13], with no significant difference in progression-free survival, which was even significantly better in the subgroup of patients older than 65 years treated with the FLO regimen. This advantage was probably due to a better safety profile of the FLO regimen with a longer median duration of treatment (two times longer in this subgroup).

Finally, trastuzumab significantly improves OS in patients with HER2 high expression [14], when added to an $\mathrm{XP}$ regimen, although this population represents less than $15 \%$ of patients with AGC, and other chemotherapy combinations have not been tested with trastuzumab.

Current standard treatments for AGC include EOX, DCF including xelox, FOLFOX, XP and some other regimens, but metastatic AGC still has a very poor prognosis with a median survival of less than 12 months in most trials. Fluoropyrimidines, platinum compounds and taxanes seem to be among the most active drugs in gastric cancer.

Four years ago, we replaced in the DCF regimen $\mathrm{P}$ by $\mathrm{O}$, $\mathrm{F}$ by LV5FU2 and used $\mathrm{D}$ at the same dose but biweekly instead of every 3 weeks, thus defining a parent protocol named TEF. TEF in fact emerged as the addition of $\mathrm{D}$ to a modified FOLFOX regimen, as we hypothesized that it might be better tolerated and as effective as DCF for patients with AGC. This TEF protocol was then adopted as first-line chemotherapy in six centers in France for patients with AGC. In the present study we assessed the efficacy and tolerability of TEF.

\section{Patients and methods}

\section{Patients}

All consecutive patients treated with the TEF regimen in six participating French centers (Hôpital Ambroise Paré, Boulogne; CLCC Val d'Aurelle, Montpellier; Hôpital Européen Georges Pompidou, Paris; CHU La Timone, Marseille; CHU St-Eloi, Montpellier; Institut Curie site René Huguenin, Saint-Cloud) from March 2008 to June 2011 were prospectively enrolled in our database. Data were collected until March 2012.

Eligibility criteria were: (1) AGC or adenocarcinoma of the gastroesophageal junction, histologically confirmed as locally advanced, local relapse, or metastatic disease, and no possibility of curative resection as assessed by each center's multidisciplinary staff including an experienced surgeon, having at least one measurable lesion. (2) No previous chemotherapy for metastatic disease. (3) Previous radiotherapy or systemic chemotherapy regimens for (neo)adjuvant treatment were allowed if all treatments were stopped at least 3 months before starting TEF. (4) An ECOG performance status of 0-2. (5) No other active malignancy.

Exclusion criteria for analysis were abnormal bone marrow, renal and hepatic function, cardiac failure, previous organ transplantation, HIV or other immunodeficiency syndromes, known hypersensitivity to FU, leucovorin, oxaliplatin, or docetaxel, patients unable to give their consent, pregnancy, breast-feeding, and non-use of contraceptives in premenopausal women.

Baseline clinical and biochemical parameters and age, ECOG performance status, and histological findings were recorded.

The study was conducted in accordance with the Declaration of Helsinki and the study protocol was reviewed and approved by the institutional review board of CHU Ambroise Paré. All participating patients allowed the use of their medical records for clinical research purposes.

\section{Treatment schedule}

The biweekly intravenous TEF regimen was given as follows: docetaxel $\left(50 \mathrm{mg} / \mathrm{m}^{2}\right)$, oxaliplatin $\left(85 \mathrm{mg} / \mathrm{m}^{2}\right)$ and leucoverin $\left(400 \mathrm{mg} / \mathrm{m}^{2}\right)$ on day 1 , followed by continuous infusion of 5 -FU for $48 \mathrm{~h}\left(2400 \mathrm{mg} / \mathrm{m}^{2}\right)$ administered every 2 weeks. Prophylactic treatments, as corticosteroids, antiemetic or growth factor, were given according to standard recommendations and the physician's assessment. Dose reduction and treatment discontinuation were performed according to toxicity, disease progression and the physician's decision. 


\section{Evaluation}

Response

The objective response was evaluated every four cycles, according to RECIST criteria v1.1 based on a chest, abdomen and pelvis CT-scan (and when indicated, an MRI), compared with a baseline CT-scan performed within 3 weeks before the first cycle of TEF.

\section{Tolerability}

Toxicity was evaluated before each cycle according to the NCI-CTC v4 scale. Physical examination, a full blood count, blood electrolytes, serum creatinine and liver function tests were checked before each cycle and we retrospectively encoded each maximal toxicity reported during the treatment. Neurotoxicity was assessed before each cycle and was retrospectively evaluated according to the NCI-CTC v4 scale.

Statistical hypothesis and analysis

As a prospective observational cohort, this study did not require sample size calculation. However, we calculated the following sample size to conclude and we stopped data collection when we achieved this sample size. Using Fleming one step design unilateral alpha type one error of $5 \%$ and power of $90 \%$ with the following hypotheses: an overall objective response rate of $25 \%$ was considered as uninteresting, and an overall objective response rate of $46 \%$ was expected; it was required to include 41 pts at the end of the study. Amongst the 41 pts, if we observed 14 $(34.1 \%)$ or less with overall objective response, treatment was considered uninteresting. If we observed 15 (36.6\%) or more than 15 patients with overall objective response, the treatment was considered promising.

The objective response rate was evaluated in the intentto-treat patients who received at least one cycle of TEF. Toxicity was analyzed in the population with at least one evaluation of tolerability after treatment. Time to progression was defined as the time elapsed from the start of TEF chemotherapy until the date of progression. Progression-free survival was defined as the time elapsed from the start of TEF chemotherapy until the date of progression or death (all causes), whichever occurred first. Surviving patients without disease progression were censored at the last follow-up date. Overall survival was defined as the time elapsed from the start of TEF until death (all causes). Surviving patients were censored at the last follow-up date. Survival curves were estimated using the Kaplan-Meier method. Median follow-up and the $95 \%$ CI were calculated with the reverse Kaplan-Meier method.

\section{Results}

Patients

Forty-one patients treated with TEF were included in this study. Their mean age was 53.5 years (range, 33-73 years). Patient characteristics are summarized in Table 1.

Thirty-seven patients had metastatic disease $(90 \%)$ and four had locally advanced disease $(10 \%)$; the primary tumor was removed in 14 patients who relapsed. Thirteen of 14 patients who relapsed received neoadjuvant and/or adjuvant chemotherapy or radiotherapy-chemotherapy

Table 1 Patient characteristics

\begin{tabular}{lc}
\hline Characteristics & $n(\%)$ \\
\hline Sex & \\
Men & $26(63.4)$ \\
Women & $15(36.6)$ \\
Age & \\
$<65$ & $35(85)$ \\
$\geq 65$ & $6(15)$ \\
ECOG performance status & \\
0 & $11(27)$ \\
1 & $24(58)$ \\
2 & $6(15)$ \\
Location of primary tumor & \\
GE junction & $17(41)$ \\
Gastric & $24(59)$ \\
Prior treatment & \\
Surgery & $14(34)$ \\
Radiotherapy & $5(12)$ \\
Perioperative chemotherapy & $13(32)$ \\
No prior treatment & $27(66)$ \\
Disease status & $10(24)$ \\
Synchronous metastatic disease & \\
Metachronous metastatic disease & $22(54)$ \\
Locally advanced & $24(58)$ \\
Local relapse & $13(32)$ \\
Location of metastases & $3(7)$ \\
Liver & $1(2)$ \\
Lymph node & \\
Peritoneum & \\
Bone & \\
Lungs & \\
More than one metastatic site & \\
Mixed type or unclassified & \\
\hline
\end{tabular}


$(n=5)$. These treatments were LV5FU2 (1/13), LV5FU2$\mathrm{P}(9 / 13), \mathrm{ECF}(1 / 13)$ or FOLFOX (2/13).

Safety

A total of 310 cycles of TEF were administered, with a mean of 7.6 cycles per patient (range, 1-21) and $90 \%$ of patients received at least four cycles $(n=37)$.

Toxicities are described in Table 2 . Grade 3 toxicity occurred in 18 patients $(45 \%)$ and grade 4 in four $(10 \%)$. There was no treatment-related death. The most common grade 3-4 toxicity was neutropenia (30\%), and febrile neutropenia occurred in three patients $(8 \%)$. Primary prophylactic G-CSF was administered to 22 patients. In this group of patients the rate of grade 3-4 neutropenia decreased to $14 \%$, and only one patient (5\%) had febrile neutropenia. In contrast, in patients who did not receive prophylactic G-CSF, grade 3-4 neutropenia was reported in nine out of 18 patients $(50 \%)$.

Other frequent grade 3-4 toxicities were asthenia $(10 \%)$ and anemia $(10 \%)$. Grade $2-3$ sensitive peripheral neuropathy was observed in $12.5 \%$ of patients. These adverse events led to dose reduction in 30 patients after a mean of 4.3 cycles; oxaliplatin had to be reduced or interrupted in 22 patients, and docetaxel in 13 patients.

Adverse events led to treatment discontinuation in 10 patients $(25 \%)$ after a mean of 7.2 cycles. The causes of

Table 2 Toxic effects according to National Cancer Institute Common Toxicity Criteria Version 4

\begin{tabular}{|c|c|c|c|}
\hline Type of toxicity & All grade $(\%)$ & Grade $3(\%)$ & Grade $4(\%)$ \\
\hline \multicolumn{4}{|l|}{ Hematologic toxicities } \\
\hline Neutropenia & $15(37.5)$ & $8(20)$ & $4(10)$ \\
\hline Febrile neutropenia $^{a}$ & $3(7.5)^{\mathrm{a}}$ & NA & NA \\
\hline Anemia & $21(52.5)$ & $4(10)$ & 0 \\
\hline Thrombocytopenia & $6(15)$ & $1(2.5)$ & 0 \\
\hline \multicolumn{4}{|c|}{ Non-hematologic toxicities } \\
\hline Neuropathy & $31(77.5)$ & $5(12.5)$ & NA \\
\hline Asthenia & $19(47.5)$ & $4(10)$ & 0 \\
\hline Edema & $3(7.5)$ & $3(7.5)$ & 0 \\
\hline Nausea-vomiting & $21(52.5)$ & $2(5)$ & 0 \\
\hline Diarrhea & $17(42.5)$ & $2(5)$ & 0 \\
\hline Mucositis & $12(30)$ & $1(2.5)$ & 0 \\
\hline Alopecia $^{\mathrm{b}}$ & $12(30)$ & $8(20)^{\mathrm{b}}$ & NA \\
\hline Allergy & $3(7.5)$ & 0 & 0 \\
\hline Ungueal toxicity & $2(5)$ & 0 & 0 \\
\hline Hand-foot syndrome & $1(2.5)$ & 0 & 0 \\
\hline All toxicity & $38(95)$ & $22(55)$ & $4(10)$ \\
\hline
\end{tabular}

interruption were cutaneous reactions considered as grade 2 allergic reactions to docetaxel (two patients), neuropathy (three patients), edema (two patients) neutropenia (two patients), and nausea/vomiting (one patient).

\section{Efficacy}

In $13(43 \%)$ of 30 patients with altered baseline performance status (PS 1: 24; PS 2: 6), PS improved during treatment (PS 1: 8; PS 2: 5).

In three patients, the radiologist was unable to evaluate the response with RECIST 1.1 criteria because of the appearance of ascitis with dissociated response of targets. Two patients died before the first CT-scan evaluation and were considered as progressive. Out of the 41 patients, 27 patients had an objective response and the intent-to-treat objective response rate was $66 \%$ [95 \% CI $(50.55 ; 78.44)]$, with two confirmed complete responses after respectively four and eight cycles (Table 3).

After a median follow-up of 27.8 months, median time to progression, progression-free survival and OS were respectively 6.5 months $\quad[95 \% \quad \mathrm{CI}=(4.9 ; \quad 10.9)]$,

Table 3 Tumor response according to RECIST 1.1 criteria

\begin{tabular}{lccc}
\hline Tumor response & Number of patients (41) & $\%$ & $95 \%$ CI \\
\hline CR & 2 & 4.9 & \\
PR & 25 & 61 & \\
CR + PR & 27 & 65.9 & $50.55 ; 78.44$ \\
SD & 6 & 14.6 & \\
PD & 5 & 12.2 & \\
NE & 3 & 7.3 &
\end{tabular}

$C R$ complete response, $P R$ partial response, $S D$ stable disease, $P D$ progression of disease, $N E$ not evaluable, $C I$ confidence interval

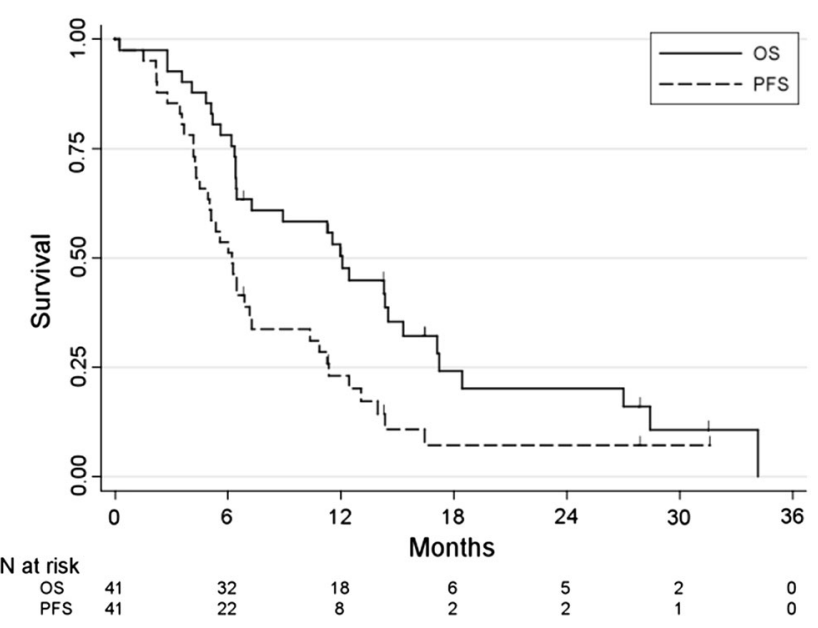

Fig. 1 Overall survival and progression-free survival 
6.3 months $\quad[95 \% \quad \mathrm{CI}=(4.5 ; 7.3)] \quad($ Fig. 1) $\quad$ and 12.1 months $[95 \% \mathrm{CI}=(6.5 ; 15.3)]$ (Fig. 1$)$.

In eight patients with linitis, the objective response rate was $75 \%$ [95 \% CI (45-105)], and median progressionfree survival and OS were respectively 6.3 months [95\% $\mathrm{CI}=(2.2 ; 11.3)]$ and 7.3 months $[95 \% \mathrm{CI}=(2.8 ; \mathrm{ND})]$.

Seven patients who were initially considered as unresectable were reconsidered for curative surgery by the multidisciplinary staff after response to TEF. Four patients underwent cytoreductive surgery and hyperthermic intraperitoneal chemotherapy for peritoneal carcinomatosis, with total gastrectomy for one patient. One patient underwent an R0 resection with total gastrectomy associated with partial hepatic resection. Two patients with locally advanced AGC had an R0 total gastrectomy with extended resection. The rate of $\mathrm{R} 0$ secondary resection was $17 \%$ (7/ 41) after TEF. After good response to TEF, two patients received a radiotherapy-chemotherapy combination to improve local control. The median OS for the nine patients who received complementary local treatment was 18.4 months $[95 \% \mathrm{CI}=(7.3, \mathrm{NA})$ months].

Thirty patients were documented as progressive on TEF treatment or discontinuated treatment due to adverse events, and 19 received FOLFIRI second line, five received other regimens, and six received best supportive care.

\section{Discussion}

There is no universal standard chemotherapy regimen in first-line treatment for $\mathrm{AGC}$, and prognosis is still very poor. Incorporation of docetaxel in cisplatin-5-FU combination regimens improved patient outcome, but at the cost of significant toxicity.

With a response rate of $65.9 \%$, and median OS of 12.1 months, this study shows that the TEF regimen administered as first-line treatment for AGC resulted in a high response rate with an acceptable toxicity profile and in specific cases allowed secondary resection. The TEF regimen may thus appear as a potential alternative to DCF.

The response rate with regimens as ECF, XP, FOLFOX or EOX is about $45 \pm 10 \%$, with a median OS between 9 and 13.3 months [10]. In the V325 trial testing DCF versus $\mathrm{CF}$, the overall response rate was $37 \%(95 \% \mathrm{CI}$ : 30.3-43.4) with DCF [10]. Within the limitations of comparing response rate from different phase II trials and the impossibility of performing cross-study comparisons with a phase III trial, we cannot conclude that TEF is superior to other docetaxel-containing regimens. However, our results compare very favorably with those obtained with chemotherapy drug combinations approved for treatment of AGC, and justify further studies. Moreover, within the limitations of a small group, TEF had some activity in linitis plastica, which is chemoresistant [15], and in eight patients with this regimen we observed six responses [corresponding to a response rate of $75 \% ; 95 \% \mathrm{CI}=(45-105)]$ and one complete response, which is seldom reported with chemotherapy in linitis plastica.

TEF appeared to be better tolerated than the DCF regimen. Grade 3-4 neutropenia occurred in $30 \%$ of patients with TEF, and rates of severe gastrointestinal toxicity were lower than $10 \%$. The rate of grade 3-4 neutropenia was $50 \%$ in patients who did not receive G-CSF and $14.7 \%$ in those who did, which is very low. In contrast, DCF resulted in a very high frequency of grade 3-4 toxicity, with severe neutropenia in $82 \%$ of cases and severe gastrointestinal disorders in $49 \%$, in the V325 study, although prophylactic G-CSF was not permitted. This underlines the value of G-CSF administration in three-drug chemotherapy for gastric cancer to decrease the risk of potential severe hematologic toxicity, especially in patients with other risk factors, such as patients older than 65 years, or with advanced disease, altered PS or nutritional status, or in patients in whom we want to increase chemotherapy doseintensity and dose-density when we hope for a secondary resection.

Several other modified DCF schemes (mDCF) were tested in an attempt to reduce toxicity. Weekly administration decreased the rate of hematological toxicity, with only $4 \%$ of grade 3-4 neutropenia [16]. However, nonhematologic toxicities were frequent with this regimen, with $23 \%$ of patients hospitalized for non-hematologic toxicity in this retrospective study by Overman et al. In a phase II trial reported by Tebbut et al. [17], with weekly mDCF there was a high rate of grade 3-4 gastrointestinal toxicity (diarrhea $22 \%$, stomatitis $18 \%$, nausea $16 \%$, asthenia $18 \%$ ) as reported earlier by Lorenzen et al. [18], who noted a similar toxicity profile.

The neurotoxicity of oxaliplatin and docetaxel is wellknown and is a major reason for stopping chemotherapy. As both drugs cause peripheral neuropathy, we were circumspect about combining them, but surprisingly neurotoxicity was not high with the TEF protocol: the $12 \%$ of grade 3 neuropathy in our study was similar to the $17 \%$ reported in the GATE study evaluating different combinations of oxaliplatin and docetaxel with or without 5-FU/ FA or capecitabine [19]. The combination of oxaliplatin and docetaxel therefore does not seem to increase neurotoxicity compared with oxaliplatin without docetaxel, as shown with FLO or EOF, with neurotoxicity in respectively 14.3 and $8.8 \%$ of cases $[12,13]$.

TEF was stopped in $25 \%$ of patients due to toxicity. This rate is high, but the rules of dose reduction and treatment stoppage were not standardized and many treatments were stopped after six or more cycles when the maximal anti-tumor effect was obtained. Allergic 
reactions, edema, or neuropathy could be managed by slowing the infusion, or by reducing or stopping a single drug, or with optimal premedications. This may be in favor of the use of the TEF regimen as induction treatment, which should be tested assuming that TEF will rapidly shrink the tumor mass and that after 2-4 months a less toxic and more convenient consolidation treatment should be instituted.

The toxicity of TEF seems to be well tolerated when TEF is used with prophylactic G-CSF, and when the doses of oxaliplatin are adapted to the neurotoxicity.

Our results are supported by other studies that have tested the combination of 5-FU, docetaxel and oxaliplatin, and by a large phase II trial in which Al-Batran et al. [20] used a regimen similar to TEF, but using 5-FU in a 24-h continuous infusion at the dose of $2600 \mathrm{mg} / \mathrm{m}^{2}$ (FLOT). The results were excellent with a response rate of $57.7 \%$, a progression-free survival of 5.2 months and an OS of 11.1 months and are confirmed by the results reported here with the TEF regimen. With the FLOT regimen, the rate of grade 3-4 neutropenia was higher (48.1\%), but patients did not receive prophylactic G-CSF. The non-hematologic toxicity profile was similar, and in particular there was very little gastrointestinal toxicity, compared with DCF or mDCF.

In the phase II randomized GATE trial, reported in abstract form, the chemotherapeutic regimen was similar to the one reported here and its therapeutic activity as well as its tolerability confirmed the good therapeutic index of the TEF regimen [19]. The response rate was $46.6 \%$ with an OS of 14.6 months, and grade 3-4 neuropathy and diarrhea were seen in respectively 17 and $11 \%$ of patients. In the two trials evaluating TEF, the efficacy/safety profile was good, justifying its use in a further phase III randomized trial.

Many questions remain, especially regarding efficacy and tolerability in elderly patients, and it has been reported that FLOT has been associated with more grade 3-4 toxicity than the oxaliplatin/5-FU combination without improvement in response rate in patients older than 70 years [21].

The present study has some limitations. The treated population was a favorable one with $85 \%$ of patients under 65 years old with a good PS (grade 0 or 1). The TEF regimen was used in this study in expert centers, with access to teams in experienced gastrointestinal oncology and surgeons able to perform hyperthermic intraperitoneal chemotherapy and liver surgery, which are not standard in this disease. Treatment modalities, as dose reductions or prophylactic use of G-CSF, were left to the discretion of the physicians in the different centers. Neurotoxicity assessment was standardized retrospectively using NCICTC v4 grading.
In conclusion, the TEF regimen appears to be at least as effective as the DCF regimen and other active regimens used for the treatment of AGC and adenocarcinoma of the gastroesophageal junction. Its efficacy in linitis, which has a poor prognosis, must be confirmed prospectively. In addition, the TEF regimen is rather well tolerated and can be used in outpatient treatment. The use of G-CSF may decrease the risk of neutropenia. TEF may also be considered in patients with overexpression of HER 2 combined with trastuzumab. The TEF regimen should now be studied in a randomized trial.

Acknowledgments The authors wish to thank the physicians and nurses from the six centers where the patients were treated and who prospectively noted the safety of the TEF regimen.

\section{References}

1. Wagner AD, Grothe W, Haerting J, Kleber G, Grothey A, Fleig WE. Chemotherapy in advanced gastric cancer: a systematic review and meta-analysis based on aggregate data. J Clin Oncol. 2006;24(18):2903-9.

2. Okines A, Verheij M, Allum W, Cunningham D, Cervantes A. Gastric cancer: ESMO clinical practice guidelines for diagnosis, treatment and follow-up. Ann Oncol. 2010;21(Suppl 5):v50-4.

3. Webb A, Cunningham D, Scarffe JH, Harper P, Norman A, Joffe JK, et al. Randomized trial comparing epirubicin, cisplatin, and fluorouracil versus fluorouracil, doxorubicin, and methotrexate in advanced esophagogastric cancer. J Clin Oncol. 1997;15(1):261-7.

4. Waters JS, Norman A, Cunningham D, Scarffe JH, Webb A, Harper $\mathrm{P}$, et al. Long-term survival after epirubicin, cisplatin and fluorouracil for gastric cancer: results of a randomized trial. Br J Cancer. 1999;80(1-2):269-72.

5. Ross P, Nicolson M, Cunningham D, Valle J, Seymour M, Harper $\mathrm{P}$, et al. Prospective randomized trial comparing mitomycin, cisplatin, and protracted venous-infusion fluorouracil (PVI 5-FU) with epirubicin, cisplatin, and PVI 5-FU in advanced esophagogastric cancer. J Clin Oncol. 2002;20(8):1996-2004.

6. Rougier P, Ducreux M, Mahjoubi M, Pignon JP, Bellefqih S, Oliveira J, et al. Efficacy of combined 5-fluorouracil and cisplatinum in advanced gastric carcinomas. A phase II trial with prognostic factor analysis. Eur J Cancer. 1994;30A(9):1263-9.

7. Mitry E, Taieb J, Artru P, Boige V, Vaillant JN, Clavero-Fabri $\mathrm{MC}$, et al. Combination of folinic acid, 5-fluorouracil bolus and infusion, and cisplatin (LV5FU2-P regimen) in patients with advanced gastric or gastroesophageal junction carcinoma. Ann Oncol. 2004;15(5):765-9.

8. Kang YK, Kang WK, Shin DB, Chen J, Xiong J, Wang J, et al. Capecitabine/cisplatin versus 5-fluorouracil/cisplatin as first-line therapy in patients with advanced gastric cancer: a randomised phase III noninferiority trial. Ann Oncol. 2009;20(4):666-73.

9. Rougier P, Oba K. Meta-analyses of randomized trials assessing the interest chemotherapy in advanced gastric cancer. J Clin Oncol. 2008;26:May 20 suppl; abstr 4563.

10. Van Cutsem E, Moiseyenko VM, Tjulandin S, Majlis A, Constenla M, Boni C, et al. Phase III study of docetaxel and cisplatin plus fluorouracil compared with cisplatin and fluorouracil as firstline therapy for advanced gastric cancer: a report of the V325 Study Group. J Clin Oncol. 2006;24(31):4991-7.

11. Ajani JA, Moiseyenko VM, Tjulandin S, Majlis A, Constenla M, Boni C, et al. Clinical benefit with docetaxel plus fluorouracil and 
cisplatin compared with cisplatin and fluorouracil in a phase III trial of advanced gastric or gastroesophageal cancer adenocarcinoma: the V-325 Study Group. J Clin Oncol. 2007;25(22): 3205-9.

12. Cunningham D, Starling N, Rao S, Iveson T, Nicolson M, Coxon $\mathrm{F}$, et al. Capecitabine and oxaliplatin for advanced esophagogastric cancer. N Engl J Med. 2008;358(1):36-46.

13. Al-Batran SE, Hartmann JT, Probst S, Schmalenberg H, Hollerbach S, Hofheinz R, et al. Phase III trial in metastatic gastroesophageal adenocarcinoma with fluorouracil, leucovorin plus either oxaliplatin or cisplatin: a study of the Arbeitsgemeinschaft Internistische Onkologie. J Clin Oncol. 2008;26(9):1435-42.

14. Bang YJ, Van Cutsem E, Feyereislova A, Chung HC, Shen L, Sawaki A, et al. Trastuzumab in combination with chemotherapy versus chemotherapy alone for treatment of HER2-positive advanced gastric or gastro-oesophageal junction cancer (ToGA): a phase 3, open-label, randomised controlled trial. Lancet. 2010;376(9742):687-97.

15. Messager M, Lefevre JH, Pichot-Delahaye V, Souadka A, Piessen G, Mariette C. The impact of perioperative chemotherapy on survival in patients with gastric signet ring cell adenocarcinoma: a multicenter comparative study. Ann Surg. 2011;254(5):684-93.

16. Overman MJ, Kazmi SM, Jhamb J, Lin E, Yao JC, Abbruzzese JL, et al. Weekly docetaxel, cisplatin, and 5-fluorouracil as initial therapy for patients with advanced gastric and esophageal cancer. Cancer. 2010;116(6):1446-53.
17. Tebbutt NC, Cummins MM, Sourjina T, Strickland A, Van Hazel G, Ganju V, et al. Randomised, non-comparative phase II study of weekly docetaxel with cisplatin and 5-fluorouracil or with capecitabine in oesophagogastric cancer: the AGITG ATTAX trial. Br J Cancer. 2010;102(3):475-81.

18. Lorenzen S, Hentrich M, Haberl C, Heinemann V, Schuster T, Seroneit T, et al. Split-dose docetaxel, cisplatin and leucovorin/ fluorouracil as first-line therapy in advanced gastric cancer and adenocarcinoma of the gastroesophageal junction: results of a phase II trial. Ann Oncol. 2007;18(10):1673-9.

19. Van Cutsem E, Boni C, Tabernero J, Massuti B, Richards DA, Prenen H, et al. Randomized phase II study (GATE study) of docetaxel plus oxaliplatin with or without fluorouracil or capecitabine in metastatic or locally recurrent gastric cancer. J Clin Oncol. 2011; suppl. abstr 4018.

20. Al-Batran SE, Hartmann JT, Hofheinz R, Homann N, Rethwisch V, Probst S, et al. Biweekly fluorouracil, leucovorin, oxaliplatin, and docetaxel (FLOT) for patients with metastatic adenocarcinoma of the stomach or esophagogastric junction: a phase II trial of the Arbeitsgemeinschaft Internistische Onkologie. Ann Oncol. 2008;19(11):1882-7.

21. Al-Batran SE, Pauligk C, Homann N, Hartmann JT, Moehler M, Probst S, et al. The feasibility of triple-drug chemotherapy combination in older adult patients with oesophagogastric cancer: a randomised trial of the Arbeitsgemeinschaft Internistische Onkologie (FLOT65 +). Eur J Cancer. 2012; Epub ahead of print. 